\title{
Analysis of gluteal subcutaneous and muscle thickness in infants and children for application to intramuscular injection, autologous fat grafting, and gluteal artery perforator flaps
}

\author{
Hyung Jun Min, Yu Jin Kim \\ Department of Plastic and Reconstructive Surgery, Gil Medical Center, Gachon University College of Medicine, Incheon, Korea
}

Background To achieve improvements in intramuscular injection, autologous fat grafting, and gluteal artery perforator flaps in infants and children, the relationships of computed tomography (CT)-determined gluteal muscle thickness (MT) and subcutaneous tissue thickness (SCT) with age and body mass index (BMI) were analyzed.

Methods Gluteal SCT and MT at ventrogluteal (VG) and dorsogluteal (DG) sites were measured in a standardized manner in 350 patients aged 0 to 6 years who had visited a tertiary hospital and had undergone abdominopelvic CT between January 2005 and December 2016. Recorded measurements were analyzed using one-way analysis of variance and stepwise multiple regression to identify the factors that were most closely related to MT and SCT.

Results Subcutaneous tissue at VG sites was thinner than at DG sites, but not significantly so in any age group. Muscles tended to be thinner at VG sites in 4 to 7-year-old, but thicker at VG sites in 1 to 3-year-old, though the differences were not significant. MT in the VG and DG regions was found to be related to age, and SCT in these regions to be related to age and BMI. Conclusions The VG and DG sites should be considered as alternatives for intramuscular injection in infants and children when the anterolateral site is problematic. In addition, considering the gluteal MT and SCT of infants and children should help produce good results in autologous fat grafting and gluteal artery perforator flap harvesting.

Keywords Injections, intramuscular / Buttocks / Surgical flaps / Child

\author{
Correspondence: Yu Jin Kim \\ Department of Plastic and \\ Reconstructive Surgery, Gil Medical \\ Center, Gachon University College of \\ Medicine, 21 Namdong-daero \\ 774beon-gil, Namdong-gu, Incheon \\ 21565, Korea \\ Tel: +82-1577-2299 \\ Fax: +82-32-460-2398 \\ E-mail: pseugene@gilhospital.com
}

Received: 11 Dec 2017 • Revised: 8 Aug 2018 - Accepted: 12 Sep 2018

pISSN: 2234-6163 • elSSN: 2234-6171 • https://doi.org/10.5999/aps.2017.01760 • Arch Plast Surg 2018;45:550-556

\section{INTRODUCTION}

Striated muscle fibers lie under the subcutaneous layer of skin and have an abundant blood supply. Therefore, complications like abscess and granuloma formation are less common after intramuscular injections than after subcutaneous injections (due to poor drainage within subcutaneous fat). Intramuscular injec- tion, as its name indicates, involves the injection of a substance directly into muscle. The needle penetrates the muscle by at least $5 \mathrm{~mm}$, and generally a needle length of 2.5 to $3.8 \mathrm{~cm}$ is used $[1,2]$. The buttocks are commonly used as a site for intramuscular injections. However, if the needle is not long enough to penetrate through the subcutaneous tissue into the muscle mass, intramuscular injections can end up being made into fat, which 
can cause granuloma or abscess formation at the injection site $[3,4]$. Accordingly, because the buttocks contain variable amounts of fat, concerns have been expressed regarding unintended injection into subcutaneous fat.

The most appropriate area for intramuscular injection remains controversial. Articles on the subject have proposed five sites for the administration of intramuscular injections: the deltoid, rectus femoris, vastus lateralis, ventrogluteal (VG), and dorsogluteal (DG) muscles [5]. For intramuscular injections in children, the anterolateral thigh and deltoid muscle are recommended. Intramuscular injection into the gluteal area is not recommended for infants, as the depth of the overlying subcutaneous layer varies and the gluteal area is small. The DG sites are at the upper outer quadrants of the buttocks, when each buttock is divided vertically and horizontally into four equal areas. In a previous study, it was suggested that the DG site be located by palpation of the greater trochanter and the posterior iliac spine and by drawing an imaginary line between the two. The DG site is lateral and superior to the midpoint of this imaginary line [6]. However, the presence of the sciatic nerve and superior gluteal artery make this site problematic. The most frequent and serious complication of gluteal injection is sciatic nerve injury. Thus, injections should be made after landmarks have been accurately and carefully identified $[7,8]$. Fig. 1 shows the best injection location to provide sufficient thickness while still avoiding injury to the sciatic nerve. In 1954, Hochsetter [9] proposed that the VG site be used for intramuscular injections; this site is located by placing the index finger of the patient's hand on the

\section{Fig. 1. Schematic of best injection location}

This schematic image shows the best injection location to provide sufficient thickness while avoiding sciatic nerve injury. The sciatic nerve passes through the lower medial quadrant. The best location is superior to a line extending between the posterior superior iliac spine and the tip of the greater trochanter.

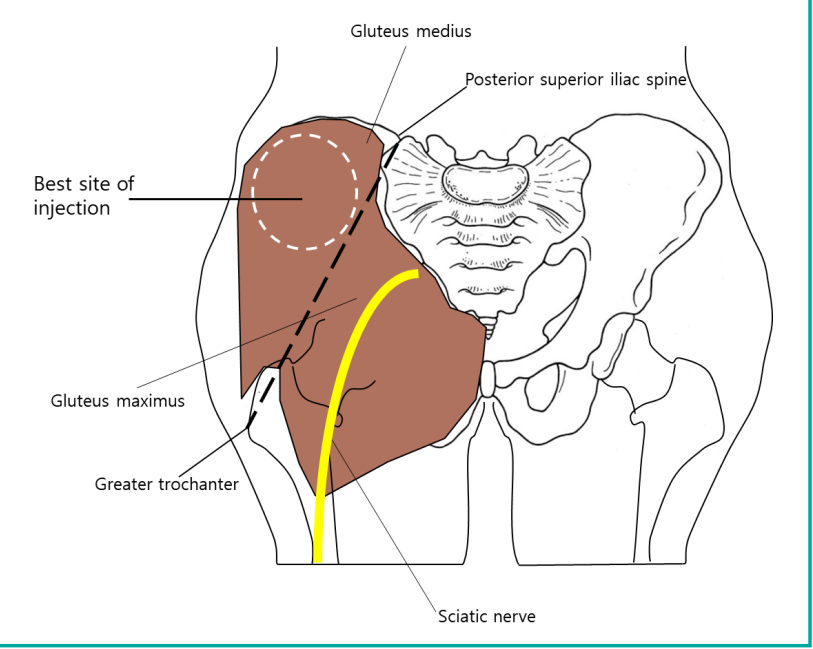

anterior superior iliac spine with the middle finger stretched dorsally towards, but below, the iliac crest or by forming a triangle with the index finger, third finger, and iliac crest [7]. The VG site consists of the gluteus medius and gluteus minimus, which have no penetrating nerves or blood vessels [10].

Previous studies have measured subcutaneous fat thickness (SCT) in the gluteal area by computed tomography (CT) to determine the proper locations for gluteal intramuscular injections in adults $[11,12]$. However, no previous study has investigated SCT and muscle thickness (MT) in the gluteal area by $\mathrm{CT}$ in children. One ultrasonographic study conducted to determine whether the VG site could be used for intramuscular injections in children found the VG site to be suitable in children under the age of 3 [13]. Data about the thickness of subcutaneous tissue and muscle in the gluteal area of children remains limited, however. One evidence-based study on site selection in infants and children was conducted with a view toward avoiding complications, such as granuloma and nerve injury, associated with inadvertent subcutaneous injection.

From a plastic surgeon's perspective, understanding soft tissue thickness and distribution in the buttock may inform the treatment of sacral pressure sores and breast reconstruction. Kim et al. [14] analyzed the soft tissue thickness of Korean adults' buttocks using CT images and a cadaveric study. Specifically in children, autologous fat grafting is used for the treatment of velopharyngeal insufficiency, facial malformations, and scar depression $[15,16]$. It is thought that considering the results of the present study at the time of autologous fat grafting could reduce preoperative planning and postoperative complications. In some pediatric patients, it is necessary to reconstruct a defect of the lower leg and the foot created by trauma or oncologic surgery. The gluteal artery perforator flap provides a thin and durable flap with minimal donor-site morbidity. This choice of flap may minimize the burden of the flap and contribute to an aesthetically pleasing appearance. In the present study, the relationships of CT-determined gluteal MT and SCT with age and body mass index (BMI) were studied in infants and children.

\section{METHODS}

\section{Subject}

We retrospectively enrolled 350 infants and children (aged 0-6 years) who had undergone abdominopelvic CT between January 2005 and December 2016. The study was initiated after obtaining IRB (GDIRB2016-346) approval from the local Ethics Committee and complied with the Declaration of Helsinki. All data used was anonymized.

The following exclusion criteria were applied: (1) injury of 
subcutaneous tissue or muscle due to surgery or trauma; (2) mass in the buttock area; (3) genetic myopathy (Duchenne muscular dystrophy, spinal muscular atrophy, or Becker muscular dystrophy); or (4) genetic skeletal disease (hypochondroplasia or achondroplasia).

\section{Data collection \\ Demographic data}

Demographic parameters (date of birth, sex, age, height, weight) were obtained for all patients. BMI was calculated from height and weight values by dividing body weight in kilograms by height in meters squared.

\section{Thicknesses of subcutaneous tissue and muscle}

MTs and SCTs were measured at the VG and DG sites of the bilateral buttocks using a digital caliper supplied with Pi-View STAR software, version 5.0.9.2 (Marotech, Infinity, Seoul, Korea). Specific CT anatomic landmarks were used to acquire consistent and reproducible measurements, as previously described by Burbridge [12]. Fig. 2 is a CT image demonstrating the locations of landmarks for DG SCT and MT measurements. The image selected was obtained $1 \mathrm{~cm}$ cranial to the caudal aspect of the sacroiliac joint using the digital calipers supplied with the PiView STAR. A line was drawn on the image along the iliac bone and a vertical line was drawn from the midpoint of this line to the skin. The lengths of the dotted lines A and B were measured. The yellow dotted line A represents DG MT. The white dotted line B represents DG SCT. Fig. 3 is a CT image showing the locations of landmarks for VG SCT and MT measurements. A line was drawn along the iliac bone, and a vertical line was then drawn from the lateral one-third point of this line to the skin.

\section{Fig. 2. CT image showing DG landmarks}

A computed tomographic (CT) image demonstrates the locations of landmarks for dorsogluteal (DG) subcutaneous tissue and muscle thickness measurements. Yellow dotted line A represents DG muscle thickness. White dotted line B represents DG subcutaneous tissue thickness.

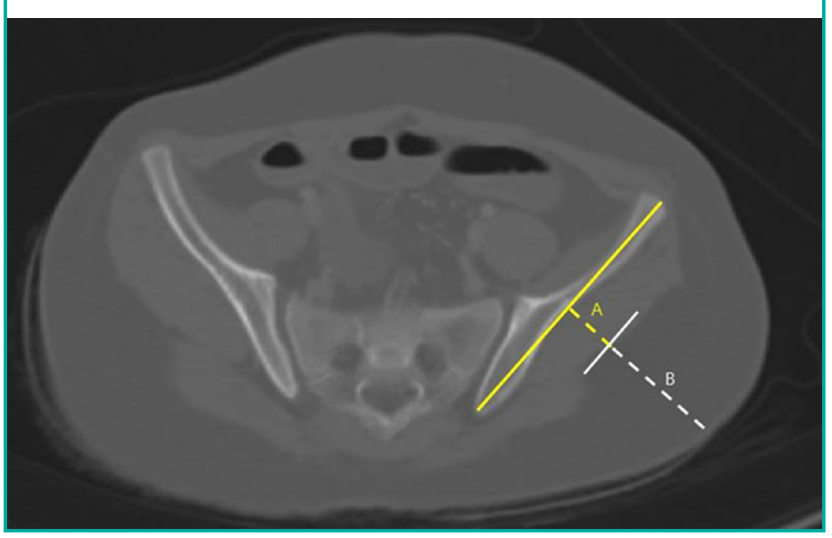

The lengths of dotted lines A and B were then measured. The yellow dotted line A represents VG MT. The white dotted line B represents VG SCT. All measurements were performed by a single investigator, and the right and left side results were averaged.

\section{Statistical analysis}

The statistical analysis was performed using SPSS for Windows version 24.0 (IBM Corp., Armonk, NY, USA). Demographic data were analyzed using descriptive statistics. One-way analysis of variance followed by the Tukey honest significant difference post hoc test was used to compare SCT and MT values in the VG and DG in different age groups. Results are presented as means \pm standard deviations, and P-values of $<0.05$ were considered to indicate statistical significance. Spearman rho correlation coefficients were used to determine the nature of the correlations between age and SCT and MT in the VG and DG areas. For this, significance was accepted for P-values $<0.05$ (two-sided test). In addition to age, we assumed that gender, height, weight, and BMI might also be correlated with MT and SCT. Therefore, a stepwise multiple regression analysis was conducted using all independent variables (age, gender, height, weight, and BMI) to identify the factors that most strongly influenced MT and SCT.

\section{RESULTS}

\section{Demographic data}

The abdominopelvic CT images of 350 patients (204 males and 146 females) were included. Patients of each year of age from 0 to 6 were equally represented ( 50 patients/group). CT had

\section{Fig. 3. CT images showing VG landmarks}

A computed tomographic (CT) image demonstrates the locations of landmarks for ventrogluteal (VG) subcutaneous tissue and muscle thickness measurements. Yellow dotted line A represents VG muscle thickness. White dotted line $B$ represents VG subcutaneous tissue thickness.

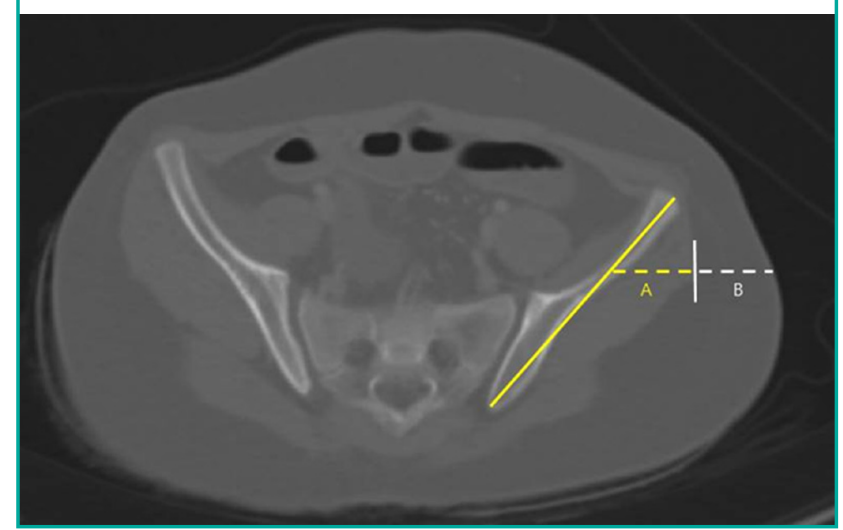


been performed for various abdominal complaints, such as pain, mesenteric ischemia, intra-abdominal mass, or infection.

\section{Subcutaneous tissue thickness}

We investigated SCT at two different DG and VG sites bilaterally. In all age groups, subcutaneous tissue was thicker at the DG sites, but the difference was not statistically significant (Table 1). In a subgroup analysis by age of SCT at the VG sites, 1-year-old had thicker gluteal fat pads $(1.78 \mathrm{~cm})$ than children in the other age groups. However, a subgroup analysis of SCT at the DG sites showed that 7-year-old had thicker gluteal fat pads $(3.31 \mathrm{~cm})$ than children in all the other age groups (Table 1). A significant positive relationship between age and SCT was observed for DG sites $(\rho=0.26, \mathrm{P}<0.01)$ (Table 2). Stepwise multiple regression of SCT at VG sites returned a multiple correlation coefficient (R) of 0.423 and an adjusted coefficient of determination $\left(\mathrm{R}^{2}\right)$ of 0.179 . Stepwise multiple regression of the SCT at DG sites returned a multiple correlation coefficient $(\mathrm{R})$ of 0.479 and an adjusted coefficient of determination $\left(R^{2}\right)$ of 0.229 . The final multiple regression equations are summarized in Table 3. The scatter plots illustrate the correlations of age with SCT and MT at the VG and DG sites with a linear regression line. A significant positive relationship between age and SCT was observed for the DG site. A significant relationship was also observed between age and MT at both the VG and DG sites (Fig. 4).

\section{Muscle thicknesses}

In this study, we measured the MT at DG and VG sites bilaterally. The MT was greater at the DG sites in children aged 4 to 7 years, but greater at the VG site in 1 to 3-year-old, although differences were not significant (Table 4). In a subgroup analysis of MT at the VG site by age, 7-year-old had thicker gluteal muscles (mean, $2.09 \mathrm{~cm}$ ) than the other age groups. Similarly, measurements of MT at the DG sites showed that, with the exception of 6-year-old, 7-year-old had thicker gluteal fat pads $(2.61 \mathrm{~cm})$ than the other children (Table 1). A significant trend was observed between age and MT at both sites of the buttocks (VG site: $\rho=0.50, P<0.01$, DG site: $\rho=0.56, P<0.01$ ) (Table 2). Step-

Table 1. General characteristics of the participants and comparisons of SCT and MT at ventrogluteal and dorsogluteal sites between age groups

\begin{tabular}{|c|c|c|c|c|c|c|c|c|c|}
\hline \multirow{2}{*}{$\begin{array}{l}\text { Age } \\
\text { (yr) }\end{array}$} & \multicolumn{2}{|c|}{ Sex } & \multirow{2}{*}{$\begin{array}{l}\text { Weight } \\
\text { (kg) }\end{array}$} & \multirow{2}{*}{$\begin{array}{l}\text { Height } \\
\text { (m) }\end{array}$} & \multirow{2}{*}{$\begin{array}{c}\text { BMI } \\
\left(\mathrm{kg} / \mathrm{m}^{2}\right)\end{array}$} & \multicolumn{2}{|c|}{ VG } & \multicolumn{2}{|c|}{ DG } \\
\hline & Male & Female & & & & SCT & MT & SCT & MT \\
\hline 0 & 37 & 13 & $7.89 \pm 2.18$ & $67.15 \pm 7.87$ & $17.24 \pm 3.02$ & $17.83 \pm 9.66^{\mathrm{a}}$ & $17.39 \pm 3.41^{\mathrm{a}}$ & $20.94 \pm 7.84^{\mathrm{a}}$ & $16.69 \pm 5.40^{\mathrm{a}}$ \\
\hline 1 & 28 & 22 & $10.70 \pm 1.96$ & $82.82 \pm 9.16$ & $15.79 \pm 3.11$ & $12.63 \pm 3.79^{b}$ & $18.87 \pm 3.56^{b}$ & $22.31 \pm 9.34^{b}$ & $17.47 \pm 4.36^{b}$ \\
\hline 2 & 21 & 29 & $12.99 \pm 1.70$ & $91.26 \pm 8.22$ & $15.81 \pm 2.50$ & $12.82 \pm 4.74^{c}$ & $19.47 \pm 2.93^{c}$ & $24.84 \pm 9.45^{c}$ & $18.50 \pm 4.53^{c}$ \\
\hline 3 & 24 & 26 & $17.12 \pm 2.57$ & $104.18 \pm 4.26$ & $15.79 \pm 2.36$ & $11.44 \pm 4.20^{d}$ & $19.08 \pm 4.39^{d}$ & $23.70 \pm 7.26^{d}$ & $21.45 \pm 6.30^{d}$ \\
\hline 4 & 37 & 13 & $20.12 \pm 4.82$ & $108.13 \pm 8.31$ & $17.19 \pm 3.56$ & $12.17 \pm 5.87^{e}$ & $12.17 \pm 5.87^{e}$ & $21.47 \pm 5.03^{e}$ & $24.35 \pm 8.91^{e}$ \\
\hline 5 & 29 & 21 & $23.47 \pm 4.46$ & $114.96 \pm 6.99$ & $17.82 \pm 3.32$ & $12.55 \pm 5.33^{\dagger}$ & $12.55 \pm 5.33^{f}$ & $22.48 \pm 5.30^{f}$ & $25.12 \pm 9.71^{\dagger}$ \\
\hline 6 & 28 & 22 & $28.22 \pm 6.56$ & $124.59 \pm 9.53$ & $18.14 \pm 3.45$ & $15.50 \pm 6.60^{9}$ & $15.59 \pm 6.60^{9}$ & $27.68 \pm 14.35^{9}$ & $33.16 \pm 11.89^{9}$ \\
\hline Total & 204 & 146 & $17.22 \pm 7.74$ & $99.01 \pm 19.98$ & $16.83 \pm 3.19$ & $13.57 \pm 6.34$ & $13.57 \pm 6.34$ & $20.92 \pm 7.34$ & $24.92 \pm 9.91$ \\
\hline \multirow[t]{2}{*}{ F } & & & & & & 7.16 & 13.21 & 8.89 & 27.41 \\
\hline & & & & & & $\begin{array}{l}(a>b, a>c, a>d \\
a>e, a>f, a>g)^{a)}\end{array}$ & $\begin{array}{l}(g>a, g>b, g>c \\
g>d, g>e, g>f)^{a)}\end{array}$ & $\begin{array}{l}(g>a, g>b, g>c \\
g>d, g>e, g>f)^{a)}\end{array}$ & $\begin{array}{l}(g>a, g>b, g>c, \\
g>d, g>e, g>f)^{a)}\end{array}$ \\
\hline P-value & & & & & & 0.01 & 0.01 & 0.01 & 0.01 \\
\hline
\end{tabular}

Table 2. Correlation of age with SCT and MT at ventrogluteal and dorsogluteal sites

\begin{tabular}{|lccc|}
\hline Variable & Site & $\rho$ & P-value \\
\hline SCT & VG & -0.058 & 0.282 \\
& DG & 0.500 & $<0.001$ \\
MT & VG & 0.262 & $<0.001$ \\
& DG & 0.560 & $<0.001$ \\
\hline
\end{tabular}

Spearman rho correlation coefficients were used to determine correlations between age and subcutaneous tissue thickness (SCT) and muscle thickness (MT) in the ventrogluteal site (VG) and dorsogluteal site (DG) areas. For this, significance was accepted for P-values $<0.05$ (2-sided test).
Table 3. Multiple regression equations using multivariate analysis

\begin{tabular}{|lcccc|}
\hline Variable & Site & Equation & $\mathrm{R}^{2}$ & $\beta$ \\
\hline SCT & VG & $y=1.537+0.853 \mathrm{X}_{1}-0.531 \mathrm{X}_{2}$ & 0.179 & 0.853 \\
& $\mathrm{DG}$ & $\mathrm{y}=0.531+1.176 \mathrm{X}_{1}+1.151 \mathrm{X}_{2}$ & 0.229 & 1.176 \\
MT & VG & $y=8.125+0.451 \mathrm{X}_{2}$ & 0.316 & 0.451 \\
& DG & $\mathrm{y}=8.869+0.326 \mathrm{X}_{2}$ & 0.320 & 0.326 \\
\hline All independent variables (age, sex, height, weight, BMI) & were entered into \\
stepwise multiple regression models. In the equation, $\mathrm{X}_{1}$ is the BMl and $\mathrm{X}_{2}$ is age. \\
BMl, body mass index; SCT, subcutaneous tissue thickness; VG, ventrogluteal \\
site; DG, dorsogluteal site; MT, muscle thickness.
\end{tabular}




\section{Fig. 4. Correlations of age with SCT and MT}

These scatter plots illustrate the correlations of age with subcutaneous tissue thickness (SCT) and muscle thickness (MT) of ventrogluteal (VG) and dorsogluteal (DG) sites with a linear regression line. A significant increasing trend in the relationship between and SCT was observed at DG sites $(\rho=0.26, P<0.01)$. Another significant trend was observed between age and MT at both sites of the buttocks (VG site: $\rho=0.50, P<0.01, D G$ site: $\rho=0.56, P<0.01)$.

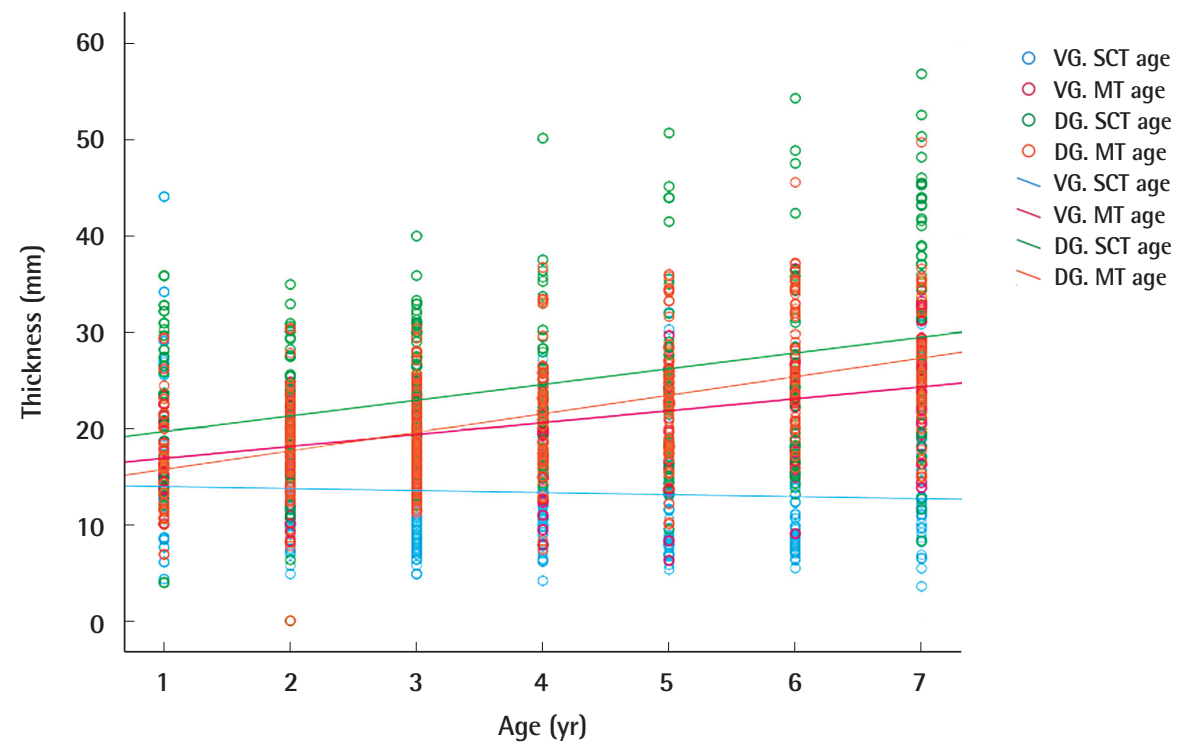

Table 4. Comparison of subcutaneous tissue and muscle thickness between ventrogluteal and dorsogluteal sites by age group

\begin{tabular}{|c|c|c|c|c|c|c|}
\hline \multirow{2}{*}{ Age (yr) } & \multicolumn{2}{|c|}{ SCT } & \multirow{2}{*}{ P-value ${ }^{\text {a) }}$} & \multicolumn{2}{|c|}{ MT } & \multirow{2}{*}{ P-value ${ }^{a)}$} \\
\hline & VG & DG & & VG & DG & \\
\hline 1 & $17.83 \pm 9.66$ & $20.94 \pm 7.84$ & 0.059 & $17.39 \pm 3.41$ & $16.69 \pm 5.40$ & 0.061 \\
\hline 2 & $12.63 \pm 3.79$ & $22.31 \pm 9.34$ & 0.064 & $18.87 \pm 3.56$ & $17.47 \pm 4.36$ & 0.079 \\
\hline 3 & $12.82 \pm 4.74$ & $24.84 \pm 9.45$ & 0.072 & $19.47 \pm 2.93$ & $18.50 \pm 4.53$ & 0.059 \\
\hline 4 & $11.44 \pm 4.20$ & $23.70 \pm 7.26$ & 0.052 & $19.08 \pm 4.39$ & $21.45 \pm 6.30$ & 0.091 \\
\hline 5 & $12.17 \pm 5.87$ & $21.47 \pm 5.03$ & 0.073 & $12.17 \pm 5.87$ & $24.35 \pm 8.91$ & 0.052 \\
\hline 6 & $12.55 \pm 5.33$ & $22.48 \pm 5.30$ & 0.087 & $12.55 \pm 5.33$ & $25.12 \pm 9.71$ & 0.076 \\
\hline 7 & $15.5 \pm 6.60$ & $27.68 \pm 14.35$ & 0.066 & $15.59 \pm 6.60$ & $33.16 \pm 11.89$ & 0.081 \\
\hline Total & $13.57 \pm 6.34$ & $20.92 \pm 7.34$ & & $13.57 \pm 6.34$ & $24.92 \pm 9.91$ & \\
\hline
\end{tabular}

wise multiple regression of MT at the VG site returned a multiple correlation coefficient $(\mathrm{R})$ of 0.565 and an adjusted coefficient of determination $\left(R^{2}\right)$ of 0.316 , and stepwise multiple regression of MT at the DG sites returned values of 0.565 and 0.320 , respectively. The final multiple regression equations are summarized in Table 3.

\section{DISCUSSION}

Several previous case studies have reported that deposition of an injected drug into subcutaneous fat may cause chronic granulomatous inflammation or granuloma. Nerve injury is another common complication following intramuscular injection. Five sites have been suggested for the administration of intramuscular injections: the deltoid, rectus femoris, vastus lateralis, VG, and DG muscles. However, the most appropriate area for intramuscular injection remains controversial. In several previous studies, the vastus lateralis muscle was recommended for infants and the deltoid for children $\geq 12$ months. Another study recommended the vastus lateralis from birth to 2 years and the deltoid muscle from age 3 years [17-19]. The vastus lateralis remains the recommended site up to the age of 3 , when the VG site becomes an alternative. The deltoid is rarely used in children aged $<6$ years and then only when the volume injected is small. The 
DG site is not used in any child that has not walked for at least 1 year, and it is strongly recommended that the DG site not be used in children aged $<6$ years. The VG area is a recommended site after the age of 3 years, and the DG site is not recommended for children $<6$ years. However, it has been shown the gluteus medius in the VG area is adequately developed for intramuscular injection, even in infants. A preliminary experimental study conducted by Hughes concluded that the VG site should be used for all intramuscular injections in children [20]. Others have reported that MTs and SCTs in the VG and anterolateral sites are similar between 2 and 18 months and have suggested that the VG site be viewed as the site of choice for intramuscular injections [21]. Furthermore, it has been reported that muscle at the VG site is adequately developed even in infants between the ages of 1 and 12 months and that the VG site is thicker than the anterolateral site in children 12 to 36 months old [13].

The present study showed that MT in the VG and DG areas was dependent on age and that SCT in these areas was related to age and BMI. In addition, the multiple regression equations for MT and SCT in the VG and DG areas could be used to determine appropriate needle lengths for intramuscular injections in children. In a previous study, BMI was found to decrease between 1 and 6 years, but to increase between 6 and 10 years due to the fat accumulation rebound phenomenon [22]. The present study showed a consistent decreasing BMI trend between the ages of 1 and 10 years (Table 1 ). The subcutaneous tissue at the VG site has been reported to be thinner than at the DG site $[23,24]$, and we also observed this tendency, albeit without significance in any age group (Table 4). Yapucu Gunes et al. [13] investigated SCT and MT of the anterolateral, deltoid, and VG areas in infants and children aged 1 to 36 months using ultrasound. Burbridge [12] used pelvic CT images to measure SCT in the gluteal region, and another study used ultrasonography to investigate SCT and MT at the gluteus [25]. We investigated SCT and MT at the DG and VG sites in children and sought to clarify the relationships between SCT and MT at the DG and VG sites. In the present study, the SCT and MT at the VG and DG sites were not significantly different in 1 to 7 -year-old. As shown in Table 2, Spearman rho correlation coefficients were used to determine the correlations between age and SCT and MT in the VG and DG areas. Our analysis revealed a significant positive relationship between age and SCT at the DG site, as well as a significant relationship between age and MT at both the DG and VG sites. However, in addition to age, we suspected that gender, height, weight, and BMI may also have been correlated with the MTs and SCTs. Therefore, a stepwise multiple regression analysis was conducted using all independent variables (age, gender, height, weight, and BMI) to identify factors that were most strongly related to MT and SCT. MT in the VG and DG areas was found to be related to age, and SCT in the VG and DG areas to age and BMI. Thus, we conclude that VG and DG sites could be considered alternatives when the anterolateral site is problematic, and that the gluteal region should be considered to reduce the risk of complications after intramuscular injection in children. Autologous fat grafting in children is used for the treatment of velopharyngeal insufficiency, facial malformations, and scar depression. For the correction of facial soft tissue asymmetry, and volume deficits in particular, autologous fat grafting and dermis-fat grafts are valuable tools. In the pediatric age group, the buttock is usually used for donor harvesting and provides the best source for sufficient donor tissue and quick harvesting. Taking into consideration the results of this study at the time of autologous fat grafting can be expected to reduce preoperative planning and postoperative complications. In some pediatric patient cases, it is necessary to reconstruct a defect of the lower leg and the foot created by trauma or oncologic surgery. Reconstruction of a defect of the posterior aspect of the leg and hindfoot requires a thin flap to minimize the burden of the flap and to achieve an aesthetically pleasing appearance. The gluteal artery perforator flap is a musculocutaneous flap that is widely used with well-developed techniques. It can provide a thin and durable flap with minimal donor-site morbidity. Considering the results of this study when reconstructing defects of the lower leg and foot in pediatric patients should minimize the burden of the flap and help achieve an aesthetically pleasing appearance. All abdominopelvic CT images were acquired in the supine position, and thus, the MT and SCT in the DG area were measured to be smaller than the actual thickness. Further study is needed to measure the thickness of the VG and DG through or using CT with 3-dimensional reconstruction.

\section{NOTES}

\section{Conflict of interest}

No potential conflict of interest relevant to this article was reported.

\section{Ethical approval}

The study was approved by the Institutional Review Board of Gil Medical Center (IRB No. GDIRB2016-346) and performed in accordance with the principles of the Declaration of Helsinki.

\section{Patient consent}

Written informed consent was waived by Institutional Review Board of Gil Medical Center. 


\section{REFERENCES}

1. Chan VO, Colville J, Persaud T, et al. Intramuscular injections into the buttocks: are they truly intramuscular? Eur J Radiol 2006;58:480-4.

2. Zaybak A, Gunes UY, Tamsel S, et al. Does obesity prevent the needle from reaching muscle in intramuscular injections? J Adv Nurs 2007;58:552-6.

3. Haramati N, Lorans R, Lutwin M, et al. Injection granulomas: intramuscle or intrafat? Arch Fam Med 1994;3:146-8.

4. Poland GA, Borrud A, Jacobson RM, et al. Determination of deltoid fat pad thickness: implications for needle length in adult immunization. JAMA 1997;277:1709-11.

5. Workman B. Safe injection techniques. Nurs Stand 1999;13: 47-53.

6. Craven RF, Hirnle CJ. Fundamentals of nursing: human health and function. 2nd ed. New York: Lippincott, 1996.

7. Kozier B, Erb G, Blais K. Techniques in clinical nursing. 4th ed. Redwood City: Addison-Wesley, 1993.

8. Rosdahl CB. Textbook of basic nursing. 6th ed. Philadelphia: Lippincott, 1995.

9. Hochsetter VA. Uber die intraglutaale injektion, ihre komplicationen und deren verhutung. Shweizerische Medizinische Wochenschrift 1954;84:1226-7.

10. Zelman S. Notes on techniques of intramuscular injection: the avoidance of needless pain and morbidity. Am J Med Sci 1961;241:563-74.

11. Joo G, Sohng KY. Gluteal subcutaneous fat thickness measured by computed tomography as an estimate of proper gluteal intramuscular injections in Korean adults. J Korean Acad Nurs 2010;40:247-54.

12. Burbridge BE. Computed tomographic measurement of gluteal subcutaneous fat thickness in reference to failure of gluteal intramuscular injections. Can Assoc Radiol J 2007; 58:72-5.

13. Yapucu Gunes U, Ceylan B, Bayındir P. Is the ventrogluteal site suitable for intramuscular injections in children under the age of three? J Adv Nurs 2016;72:127-34.

14. Kim DY, Choi HN, Park JH, et al. Regional analysis of soft tissue thickness on Korean buttocks and application to fasciocutaneous flap design. Arch Plast Surg 2014;41:133-9.

15. Bishop A, Hong P, Bezuhly M. Autologous fat grafting for the treatment of velopharyngeal insufficiency: state of the art. J Plast Reconstr Aesthet Surg 2014;67:1-8.

16. Guibert M, Franchi G, Ansari E, et al. Fat graft transfer in children's facial malformations: a prospective three-dimensional evaluation. J Plast Reconstr Aesthet Surg 2013;66: 799-804.

17. Schechter NL, Zempsky WT, Cohen LL, et al. Pain reduction during pediatric immunizations: evidence-based review and recommendations. Pediatrics 2007;119:e1184-98.

18. Lippert WC, Wall EJ. Optimal intramuscular needle-penetration depth. Pediatrics 2008;122:e556-63.

19. Rishovd A. Pediatric intramuscular injections: guidelines for best practice. MCN Am J Matern Child Nurs 2014;39:10712.

20. Hughes WT. Complication resulting from an intramuscular injection. J Pediatr 1967;70:1011-3.

21. Cook IF, Murtagh J. Ventrogluteal area: a suitable site for intramuscular vaccination of infants and toddlers. Vaccine 2006;24:2403-8.

22. Wells JC. A Hattori chart analysis of body mass index in infants and children. Int J Obes Relat Metab Disord 2000;24: 325-9.

23. Rodger MA, King L. Drawing up and administering intramuscular injections: a review of the literature. J Adv Nurs 2000;31:574-82.

24. Gunes UY, Zaybak A, Tamsel S. The examination of the reliability of the method used in identifying of ventrogluteal site. J Cumhur Univ Sch Nurs 2008;12:1-8.

25. Lo Presti D, Ingegnosi C, Strauss K. Skin and subcutaneous thickness at injecting sites in children with diabetes: ultrasound findings and recommendations for giving injection. Pediatr Diabetes 2012;13:525-33. 